\title{
Total Esophagogastric Separation in Neurologically Impaired Children: Outcome and Efficacy
}

\author{
Rady Awad Elsherbini \\ Assistant Professor of Pediatric Surgery, Mansoura University, Mansoura, Egypt \\ Email: radyawad72@yahoo.com
}

How to cite this paper: Elsherbini, R.A. (2019) Total Esophagogastric Separation in Neurologically Impaired Children: Outcome and Efficacy. Open Journal of Pediatrics, 9, 126-132.

https://doi.org/10.4236/ojped.2019.92013

Received: March 25, 2019

Accepted: May 10, 2019

Published: May 13, 2019

Copyright ( 2019 by author(s) and Scientific Research Publishing Inc. This work is licensed under the Creative Commons Attribution International License (CC BY 4.0).

http://creativecommons.org/licenses/by/4.0/

Open Access

\begin{abstract}
Background: Gastroesophageal reflux disease (GERD) is one of the most clinical disorders affecting the gastrointestinal tract of neurologically impaired children (NIC). The reported incidence of GERD in NIC ranges from $15 \%$ to $75 \%$. For a long time Nissen fundoplication became the first choice for management with some complications. For this reason, and as an alternative to repeated fundoplications, total esophagogastric separation (TEGS) became popular in treating these cases. Even some studies consider TEGS as a primary approach for GERD in NIC. Purpose: The aim of the study was to assess the efficacy and outcome of total esophagogastric separation in cases of severe gastroesophageal reflux in neurologically impaired children. Materials and Methods: Medical records of 11 patients who underwent total esophagogastric separation (TEGS) with Roux en Y anastomosis for recurrent GERD in our institute in pediatric surgery unit, Medina children hospital, KSA, were reviewed retrospectively. Selective criteria included all cases of recurrent GERD in NIC after fundoplications. TEGS was not performed in any case as a primary procedure. Results: Our study included eleven cases, seven males and four females. Their ages ranged from 6 to 14 years. All of them were neurologically impaired, with difficulty to take oral feeds and all cases had GERD or its related complications preoperatively. Median operating time was 5 hours ranging from 3.5 to 7 hours with a median blood loss of $200 \mathrm{ml}$. Hospital stay ranged from 5 to 17 days with a median of 9 days and median ICU stay was 3.5 days. Complications occurred in four cases (36.3\%) with no mortality. Three cases needed reoperations (27.2\%). Two cases (18.2\%) developed hiatus hernia and were operated between 8 and 10 months postoperatively. Two cases (18.2\%) develop adhesive intestinal obstruction who underwent conservative management and passed smoothly. Conclusion: TEGS, is considered as a safe, effective and reliable operation for treating cases of
\end{abstract}


recurrent GERD after fundoplications in neurologically impaired children especially those who are dependent on enteral tube feeding.

\section{Keywords}

Total Esophagogastric Separation, Gastro-Esophageal Reflux, Nissen Fundoplication

\section{Introduction}

Gastroesophageal reflux disease (GERD) is a common problem in neurologically impaired children (NIC). It has been recognized more frequently because of an increased awareness of the condition and the more sophisticated diagnostic techniques that have been developed for both identifying and quantifying the disorder. The management of children with GERD initially begins with nonoperative measures that often result in resolution of symptoms. The goals of medical management include lifestyle modifications and acid-suppressive medications to improve the transit of gastric contents [1].

Surgical management of GERD typically becomes necessary in the presence of GER complications and/or failed medical therapy. There are major debates about which operation is reliable. Antireflux procedures are satisfactory in treating these cases. However, the recurrence rate is considering higher in this group of patients with problems of vomiting, tube feeding intolerance, aspiration pneumonia and failure to thrive [2]. Repeated fundoplication carries a higher recurrence rate in addition to major problems (adhesions, bleeding and sepsis) [3].

Total Esophagogastric Separation (TEGS) with Roux en Y anastomosis is used to treat these recurrence conditions with good results. Even, it became the primary option in treating these cases [4]. Our aim is to assess the efficacy of TEGS for treating cases of recurrent gastroesophageal reflux after fundoplication in neurologically impaired children.

\section{Patients and Methods}

Medical records of 11 patients who underwent total esophagogastric separation (TEGS) with Roux en Y anastomosis for recurrent GERD in our institute in pediatric surgery unit, Medina children hospital, KSA during the period between Feb. 2011 to March 2018 were reviewed retrospectively. Selective criteria included all cases of recurrent GERD in NIC after fundoplications. TEGS was not performed in any case as a primary procedure. Their ages range between $6-14$ years. Seven cases did TEGS for recurrent GERD symptoms after three fundoplications in three cases and two fundoplications in four cases. The recurrence of GERD was assessed clinically and radiologically. Clinical assessment was done by following cases of previous fundoplications as regarding improvement of 
body weight, signs of malnutrition, feeding tube problems, in addition to recurrent attacks of respiratory tract infections. Upper gastro-intestinal contrast studies were carried out to diagnose cases of recurrent reflux and give an idea about wrap strength (Figure 1 and Figure 2). Milk scan was needed for confirmation of GERD induced respiratory problems.

The remaining four cases were operated by TEGS for recurrent attacks of aspiration pneumonias related to GERD after two fundoplications in three cases and one fundoplication in one case. The operation was performed via a midline incision, the gastroesophageal junction and the previous wrap were identified and taken down. Stapler was used to divide the esophagus with 4 centimeters from the cardiac junction which is closed in single layer. A 25 centimeters roux en $\mathrm{Y}$ jeujenal limb was being created and brought in retro colic, retro gastric way with a single layer end to end anastomosis with the esophageal end over a nasogastric tube. Pyloroplasty was performed as a gastric emptying procedure in all cases. A gastrostomy tube was refashioned with proper sizes for feeding in all cases. All cases were shifted to the intensive care unit for a period of 7 to 14 days. Gastrostomy feeding was allowed, teached to the family then patients were discharged home on regular follow up. Review of our cases included the presenting symptoms, operative time, hospital stay and postoperative complications. On follow up visits clinical assessment included improvement in body weight, respiratory condition, feeding tube problems, and family satisfaction. Contrast study was not done postoperatively, unless indicated.

\section{Results}

Our study included eleven cases, seven males and four females. Their ages ranged between 6 - 14 years. All of them were neurologically impaired, with difficulty to take oral feeds and all cases had GERD or its related complications preoperatively. We depend on upper GIT contrast study, $24 \mathrm{hr} \mathrm{Ph}$ monitoring, and milk scan in diagnosing GERD and its related complications. Seven cases did TEGS for recurrent GERD after three fundoplications in three cases and two fundoplications in four cases. The remaining four cases were operated by TEGS for recurrent attacks of aspiration pneumonias related to GERD after two fundoplications in three cases and one fundoplication in one case. Median operating time was 5 hours range between 3.5 to 7 hours with a median blood loss of 200 $\mathrm{ml}$ which was related to the severity of adhesions from previous operations. Hospital stay ranged from 5 to 17 days with a median of 9 days and median ICU stay was 3.5 days ranged from 1 to 12 days. Patients were on the ventilation for a median of 4 days. Upper GIT contrast was routinely done in the period between 30 to 90 days postoperatively for ruling out recurrent problems. Complications occurred in four cases (36.3\%) with no mortality (Table 1). Three cases needed reoperations (27.2\%). Two cases (18.2\%) developed hiatus hernia and were operated between 8 to 10 months postoperatively. Revision of the esophagojejunal anastomosis was done in one case $(9.1 \%)$ due to leak from it. The leak was dis- 
covered on the fifth day postoperatively, patient was explored and did fine after that. Two cases (18.2\%) develop adhesive intestinal obstruction who underwent conservative management and passed smoothly.

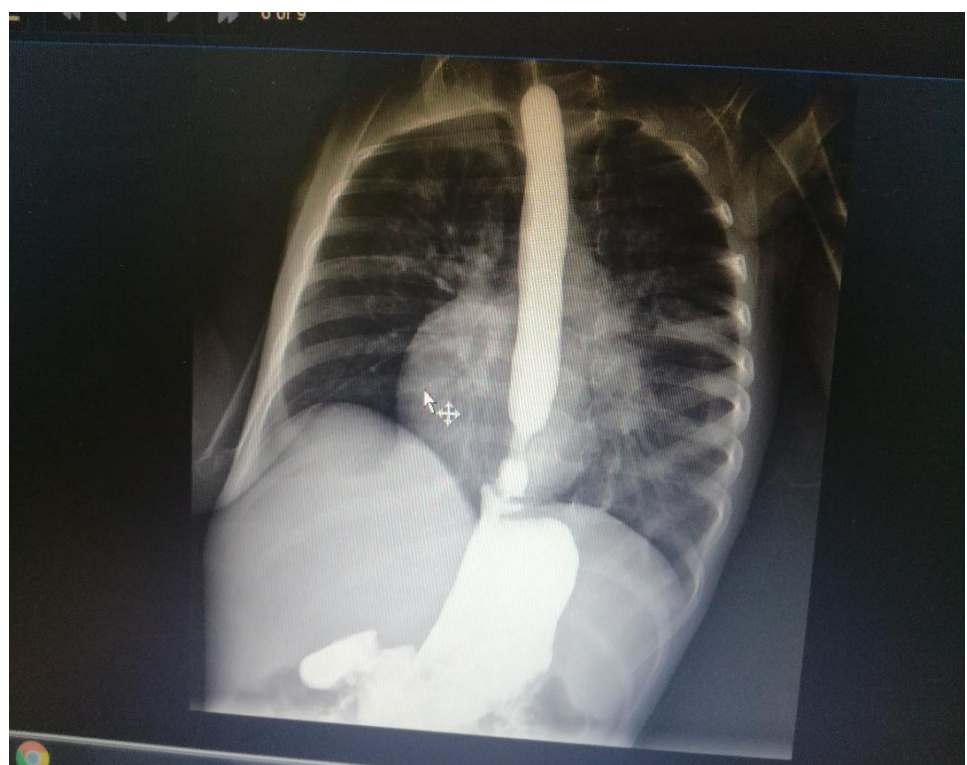

Figure 1. Severe GERD with hiatus hernia.

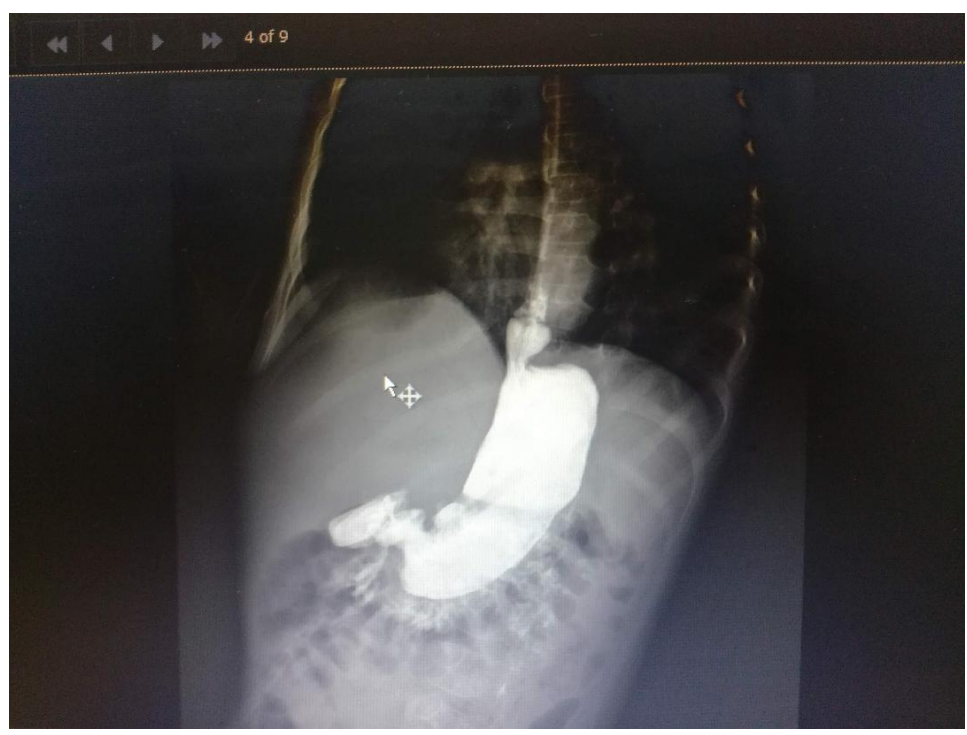

Figure 2. Wrap disruption in failed Nissen fundoplication.

Table 1. Postoperative complications in the postoperative period and on follow up visits.

\begin{tabular}{ccc}
\hline Complications & Number 0f cases (4 cases) & Percentage (36.3\%) \\
\hline Hiatus hernia & 2 & $18.2 \%$ \\
$\begin{array}{c}\text { Anastomotic leak } \\
\text { and wound infection } \\
\text { Adhesive intestinal } \\
\text { obstruction }\end{array}$ & 1 & $9.1 \%$ \\
\hline
\end{tabular}




\section{Discussion}

Gastroesophageal reflux disease (GERD) is one of the most frequent symptomatic clinical disorders affecting the gastrointestinal tract of infants and children. It has been recognized more frequently because of an increased awareness of the condition and also because of the more sophisticated diagnostic techniques that have been developed for both identifying and quantifying the disorder. The most frequent complications of GERD in childhood are failure to thrive, recurrent bronchitis or pneumonia caused by repeated pulmonary aspiration of gastric fluid and esophagitis [5].

Neurologically impaired children are at high risk for having symptomatic GERD, particularly if nasogastric or gastrostomy feedings are necessary because of delayed gastric emptying and neuromuscular incoordination. The most helpful test for diagnosing and quantifying GERD in childhood are contrast studies of the gastrointestinal tract, the 24-hour esophageal $\mathrm{pH}$ monitoring study and milk scan. Surgical management of GERD becomes necessary in presence of GERD complications and/or failed medical therapy [6].

Antireflux procedures are usually performed to eradicate the reflux of gastric contents into the esophagus which should control GERD related symptoms, prevent complications, and permit adequate caloric intake to achieve growth. This is achieved by reestablishing the gastroesophageal barrier through creation of a partial or complete valve mechanism at the gastroesophageal junction (fundoplication). Currently, laparoscopic antireflux procedures have replaced the open approach to become the primary surgical approach for the treatment of GERD [7].

Neurologically impaired children (NIC) often have pharyngeal neuromuscular incoordination and difficulty with swallowing, severe gastroesophageal reflux (GER), recurrent respiratory infections, and, consequently, severe malnutrition [8]. The reported incidence of GER in NIC ranges from $15 \%$ to $75 \%$. Despite the wide variety of procedures advocated, surgical construction of a Nissen fundoplication with a concomitant feeding gastrostomy has evolved rapidly as the definitive therapy for persistently symptomatic GER disease in NIC refractory to medical treatment [9].

In the literature, several reports have been published reporting the association of fundoplication with a $7 \%$ to $35 \%$ recurrence of reflux that required additional surgery in NIC. This failure rate may be caused by wrap failure from repeated retching, gagging, and seizures. Repeat fundoplications in this situation have a higher recurrence rate (25\%) as well as entailing a higher risk of complications [10].

There are many operations described as alternatives including placement of gastrojejunal feeding and Roux-en-y jejunostomy tube feeding and redo fundoplications. Insertion of feeding tubes can solve the problem of feeding intolerance, but cannot improve the issue of emesis [11].

The first surgery of total esophagogastric separation (TEGS) was described by 
Bianki in 1997 and he reported good results with this technique [12]. Another further studies with good results also were published, even they recommended TEGS as a primary operation for cases of GERD induced recurrent pneumonias and feeding intolerance. However these results still in need for more scientific confirmation and large comparative studies with primary fundoplications [13] [14].

In our series complications occurred in four cases $(36.3 \%)$ with no mortality. These results are higher than Tanaka Y. et al. [15] (17.2\%) and Gatti C, et al., [16] (15.5\%). This is explained by our low number of cases, although we have no mortality cases. Also, these studies included primary cases of GERD, while our study included all cases of redo surgery after fundoplications.

In our series, TEGS provided a reasonable alternative to redo antireflux procedures and was well tolerated. Some limitations of the study were our low number of patients, some difficulties in follow up, and no cases of primary TEGS. Because of this, we still believe that a fundoplication remains the first choice in the surgical treatment of GERD in NIC. And, we advise TEGS in those children with recurrent GERD and neurologic impairment especially those who are dependent on enteral tube feeding.

\section{Conclusion}

In conclusion, TEGS, is considered as a safe, effective and reliable operation for treating cases of recurrent GERD after fundoplication in neurologically impaired children.

\section{Conflicts of Interest}

The author declares no conflicts of interest regarding the publication of this paper.

\section{References}

[1] Fonkalsrud, E.W. and Ament, M.E. (1996) Gastroesophageal Reflux in Childhood. Current Problems in Surgery, 33, 3-70. https://doi.org/10.1016/S0011-3840(96)80012-8

[2] Jancelewich, T., Monica, E., Lope, A., et al. (2016) Surgical Management of Gastroesophagcal Reflux Disease in Children: A Systematic Review. Journal of Pediatric Surgery, 52, 1228-1238. https://doi.org/10.1016/j.jpedsurg.2016.09.072

[3] Subramanian, R. and Dickson, A.P. (2000) Long Term Outcome of Boix-Ochoa and Nissen Fundoplication in Normal and Neurologically Impaired Children. Journal of Pediatric Surgery, 35, 1214-1216. https://doi.org/10.1053/jpsu.2000.8729

[4] Molinarof, B.E., Cerchia, E., et al. (2014) Esophagogastric Dissociation Reduces the Re-Operation Rate for Persistent Gastroesophageal Reflux in Severely Neurologically Impaired Children. Pediatric Surgery International, 30, 997-1001. https://doi.org/10.1007/s00383-014-3584-9

[5] Hope, T., Jackson, I. and Timothy, D. (2013) Surgical Management of Pediatric Gastroesophageal Reflux Disease. Gastroenterology Research and Practice, 2013, Article ID: 863527. https://doi.org/10.1155/2013/863527 
[6] Lall, A., Morabito, A., Dall, O., et al. (2006) Total Oesophagogastric Dissociation: Experience in 2 Centres. Journal of Pediatric Surgery, 41, 342-346. https://doi.org/10.1016/j.jpedsurg.2005.11.010

[7] Hatah, K.F., Daily, M.F. and Christensen, B.T. (2004) Failed Fundoplication. The American Journal of Surgery, 188, 786-791. https://doi.org/10.1016/j.amjsurg.2004.08.062

[8] Fonkalsrud, E.W., Ashcraft, K.W., Coran, A.G., et al. (1998) Surgical Treatment of Gastroesophageal Reflux in Children: A Combined Hospital Study of 7467 Patients. The Journal of Pediatrics, 101, 419-422. https://doi.org/10.1542/peds.101.3.419

[9] Vane, D.W., Harmel, R.P., King, D.R., et al. (1985) The Effectiveness of Nissen Fundoplication in Neurologically Impaired Children with Gastroesophageal Reflux. Surgery, 98, 662-666.

[10] Smith, C.D., Mcclusky, D.A., Rajad, M.A., et al. (2005) When Fundoplication Fails: Redo? Annals of Surgery, 24, 861-869. https://doi.org/10.1097/01.sla.0000165198.29398.4b

[11] Islam, S., Teitelbaum, D.H., Buntain, W.L. and Hirschl, R.B. (2004) Esophagogastric Separation for Failed Fundoplication in Neurologically Impaired Children. Journal of Pediatric Surgery, 39, 287-289. https://doi.org/10.1016/j.jpedsurg.2003.11.035

[12] Bianchi, A. (1997) Total Esophagogastric Dissociation: An Alternative Approach. Journal of Pediatric Surgery, 32, 1291-1294. https://doi.org/10.1016/S0022-3468(97)90304-3

[13] Lall, A., Morabito, A. and Bianchi, A. (2006) Total Gastric Dissociation (TGD) in Difficult Clinical Situations. European Journal of Pediatric Surgery, 16, 396-398. https://doi.org/10.1055/s-2006-924731

[14] Chhabra, S., Nedea, A.M., Kauffman, I. and Morabito, A. (2017) Total Esophagogastric Dissociation: Single Center Experience. Journal of Pediatric Surgery, 52, 260-263. https://doi.org/10.1016/j.jpedsurg.2016.11.018

[15] Tanaka, Y., Tainaka, T. and Unchida, H. (2018) Indications for Total Esophagogastric Dissociations in Children with Gastroesophageal Disease. Surgery Today, 48, 971-977. https://doi.org/10.1007/s00595-018-1636-9

[16] Gatti, C., Abriola, F., Villa, M., et al. (2001) Esophagogastric Dissociation versus Fundoplication: Which Is Best for Severely Neurologically Impaired Children? Journal of Pediatric Surgery, 36, 677-680. https://doi.org/10.1053/jpsu.2001.22935 